Supporting Information for

\title{
Mortality Risk Associated with Short-term Exposure to Particulate Matter in China: Estimating Error and Implication
}

Hao Wang ${ }^{1,2 \#}$, Peng Yin ${ }^{3 \#}$, Wenhong Fan ${ }^{1,2}$, Ying Wang ${ }^{1,2}$, Zhaomin Dong ${ }^{1,2^{*}}$, Qihong Deng ${ }^{4,5}$, Maigeng Zhou ${ }^{3}$

1, School of Space and Environment, Beihang University, Beijing, China

2, Beijing Advanced Innovation Center for Big Data-Based Precision Medicine, Beihang University, Beijing, China

${ }^{3}$, National Center for Chronic and Noncommunicable Disease Control and Prevention, Chinese Center for Disease Control and Prevention, Beijing, China

${ }^{4}$, School of Energy Science and Engineering, Central South University, Changsha 410083, China;

5, XiangYa School of Public Health, Central South University, Changsha 410078, China.

\#. H.W. and P.Y. contributed equally to this work.

${ }^{*}$ Corresponding author:

Correspondence to Dr. Zhaomin Dong, Beijing Advanced Innovation Center for Big DataBased Precision Medicine, Beihang University, Beijing, 100191, China dongzm@buaa.edu.cn; chaomi 87@163.com

Supporting information includes 25 pages, 19 figures, 5 tables. 


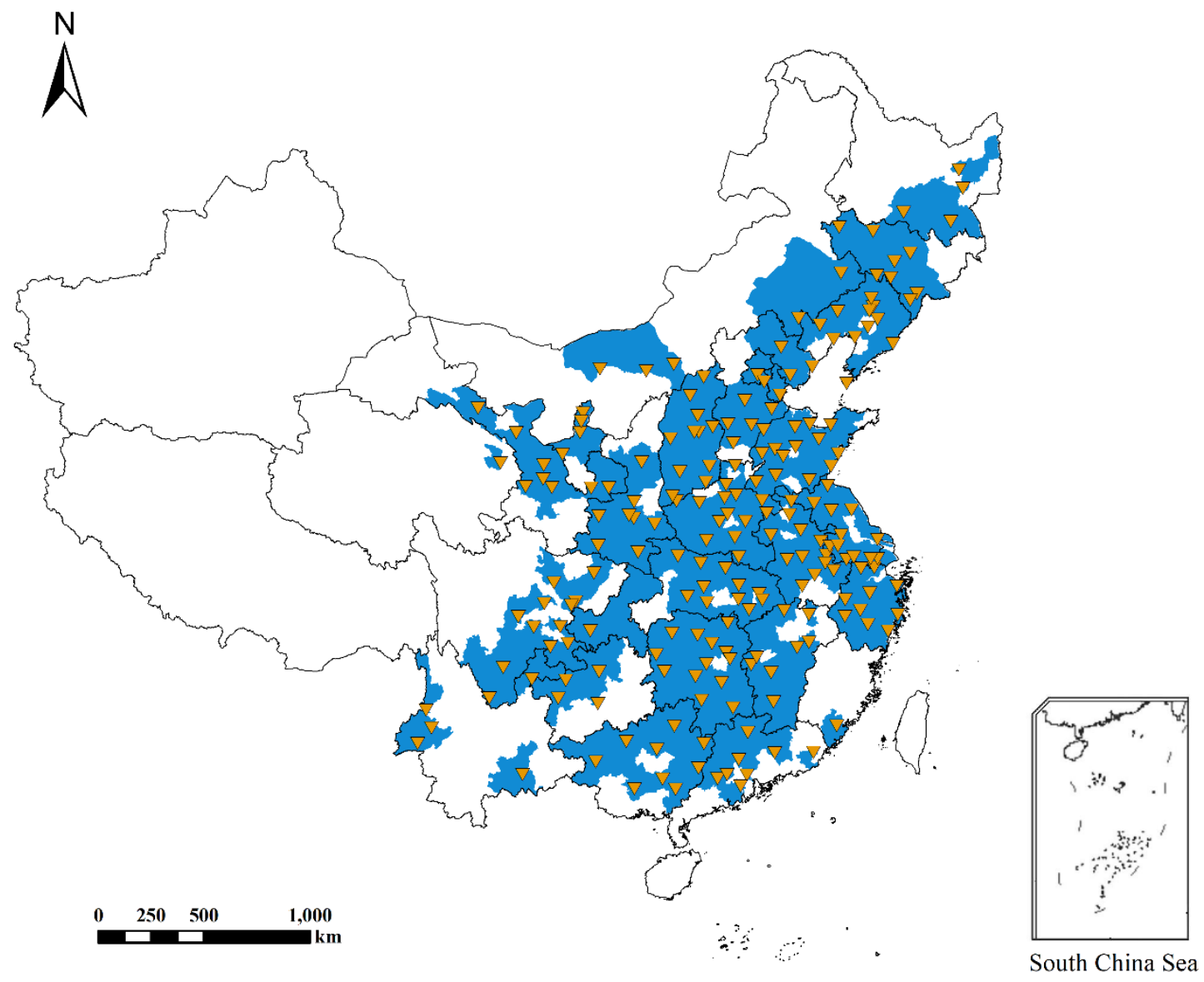

Figure S1. The administrative division of 205 cities selected in this study. The flowchart for city selection was detailed in Figure S2. 


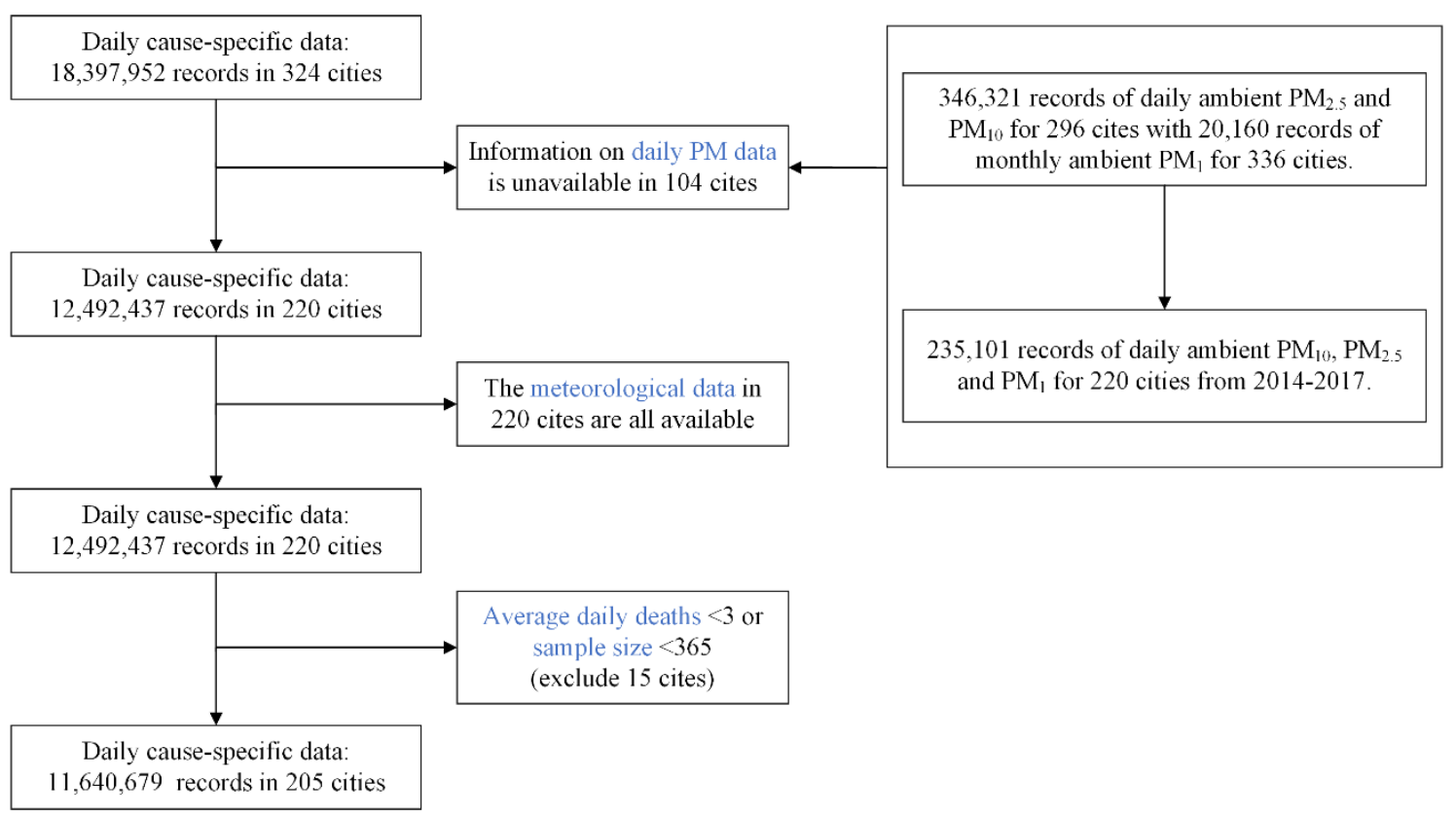

Figure S2. The flowchart for city selection. 


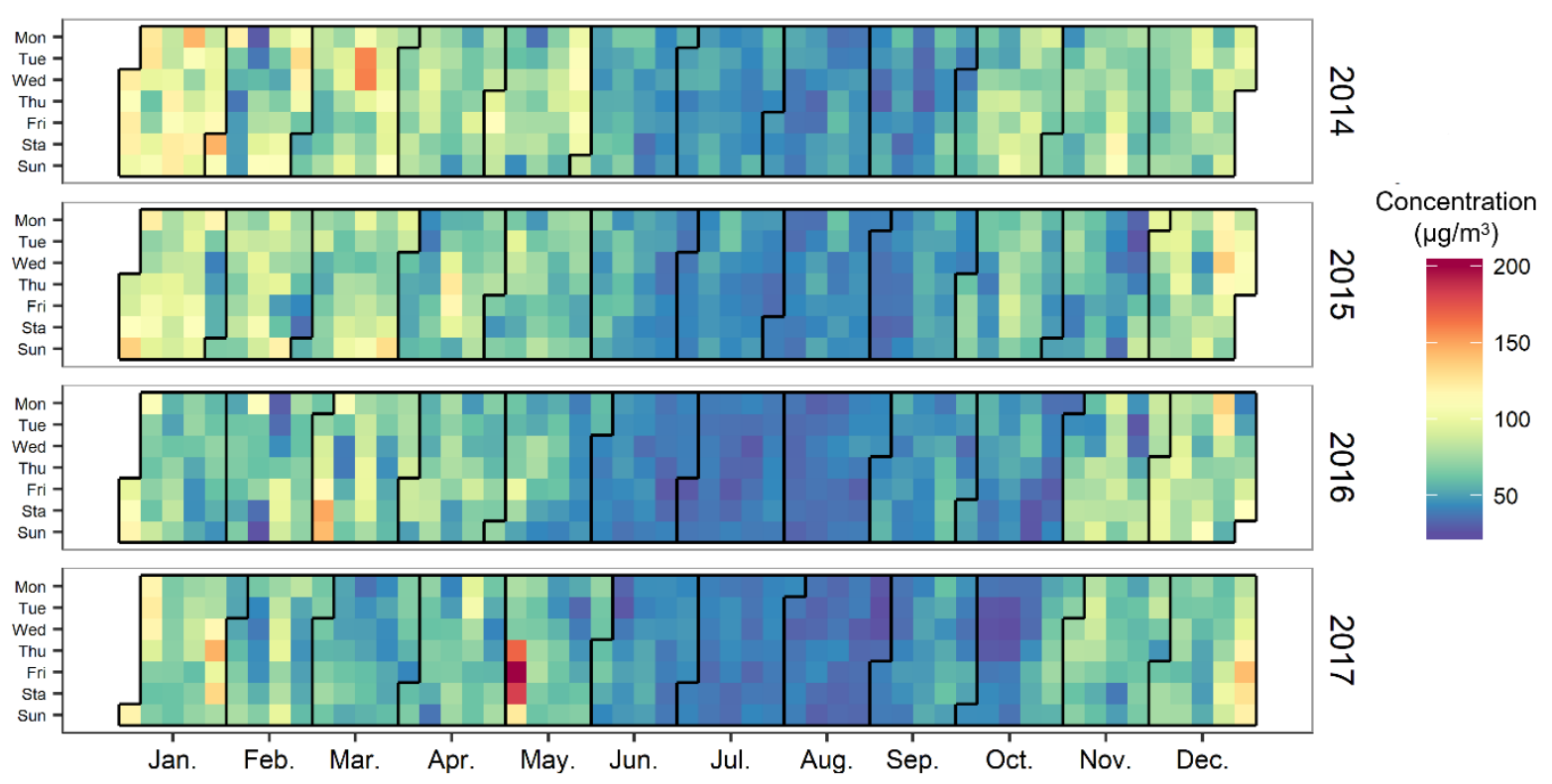

Figure S3. The daily average concentration of total deposited particulate matter in head, tracheobronchial, and pulmonary regions for the general population during 2014-2017, China. 


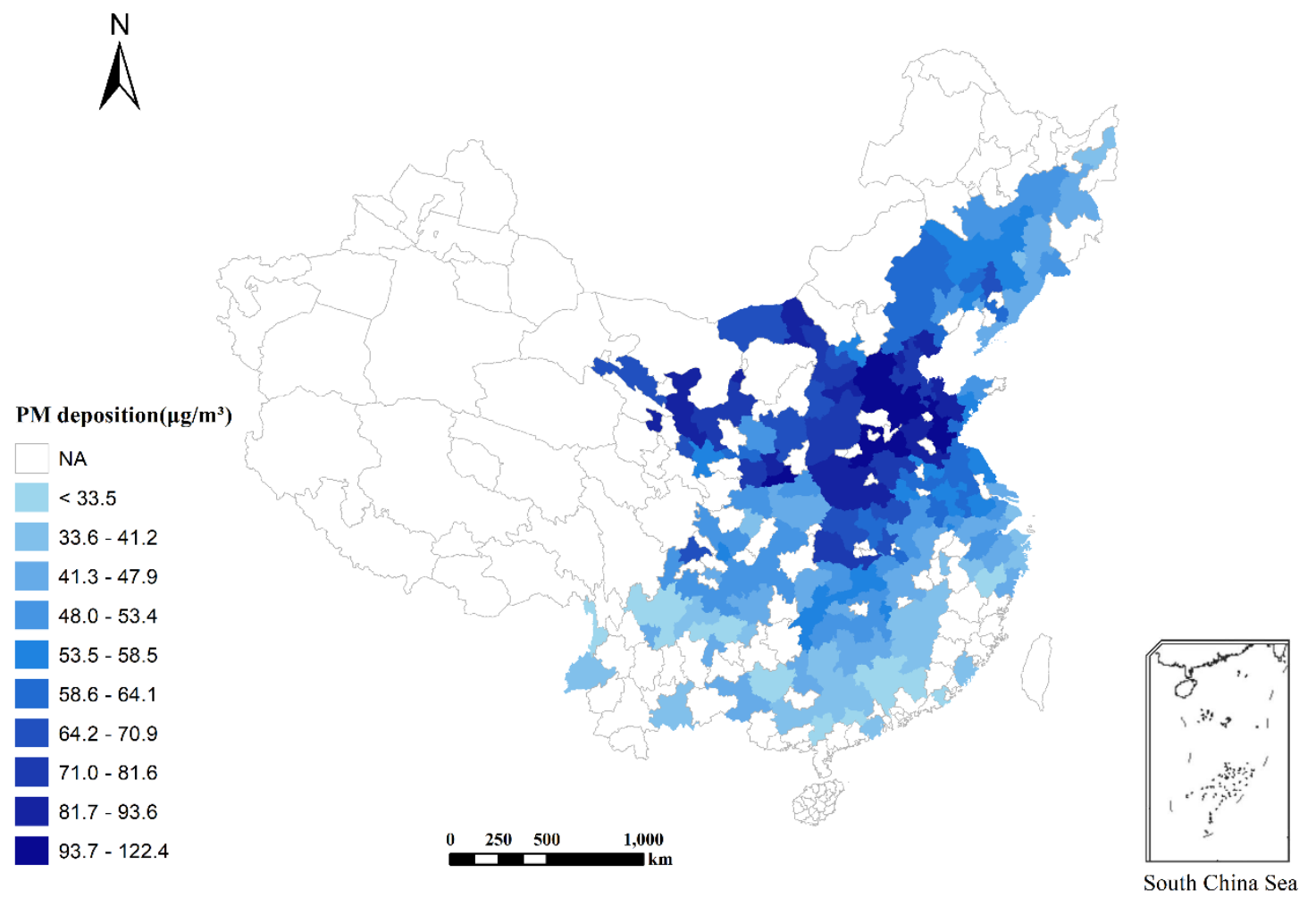

Figure S4. The annual average concentrations of total deposited particulate matter in 205 cities, China. NA: not available. 

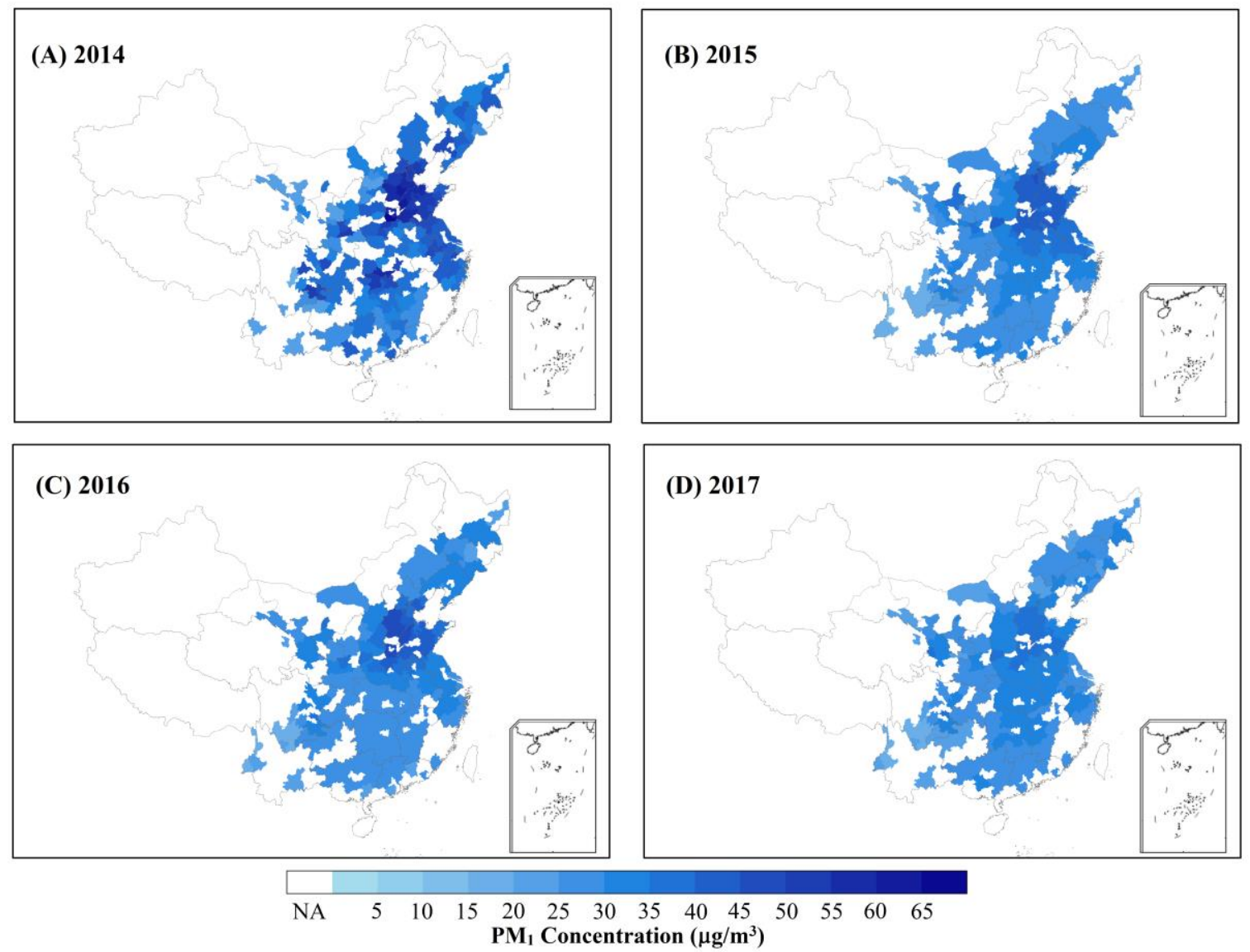

Figure S5. The daily average concentrations of $\mathrm{PM}_{1}$ in 205 cities from 2014 to 2017, China. NA: not available. 

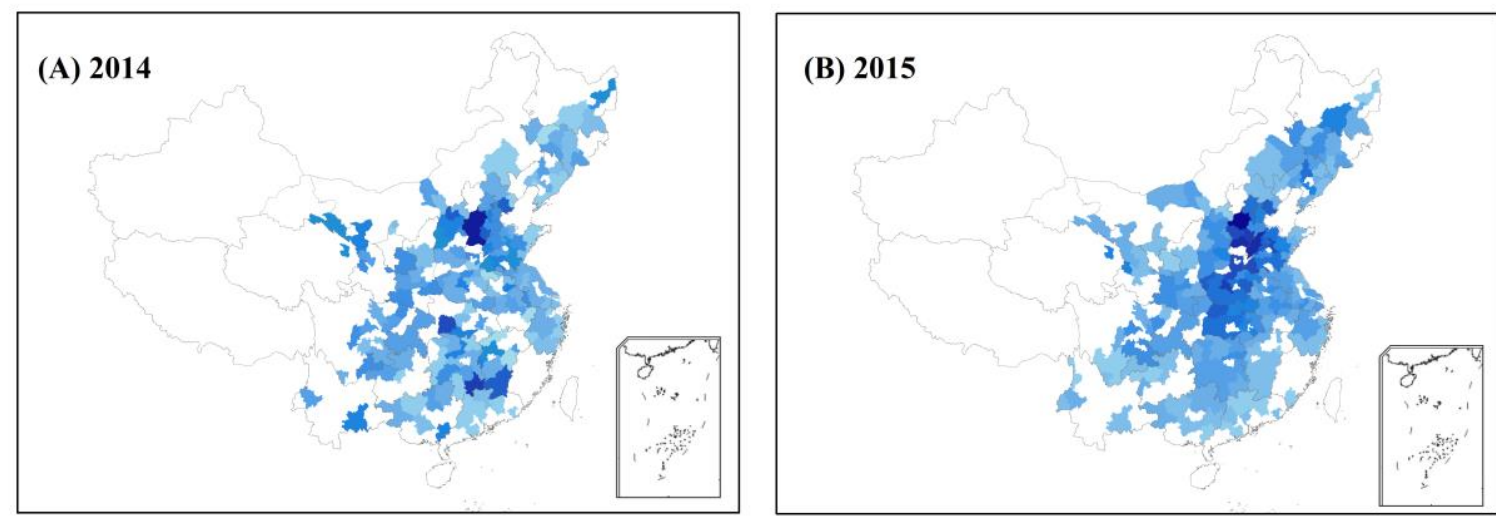

(C) 2016

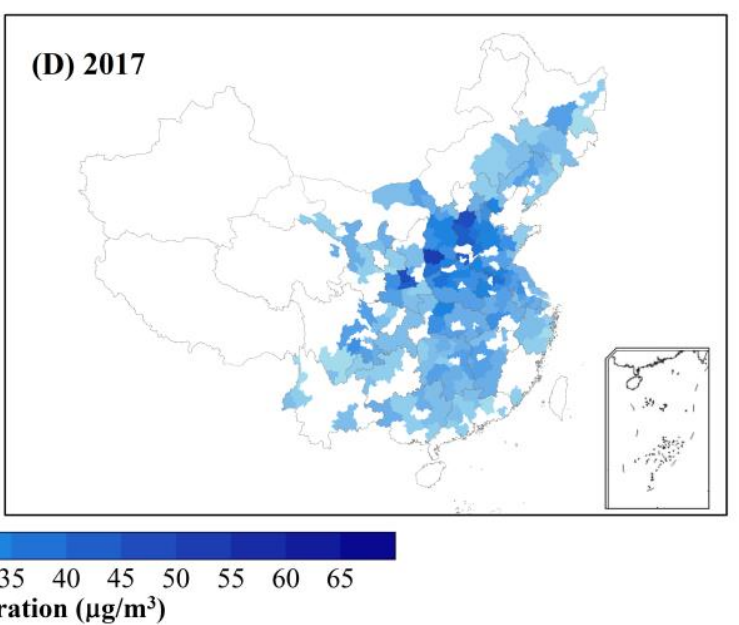

Figure S6. The daily average concentrations of $\mathrm{PM}_{1-2.5}$ in 205 cities from 2014 to 2017, China. NA: not available. 

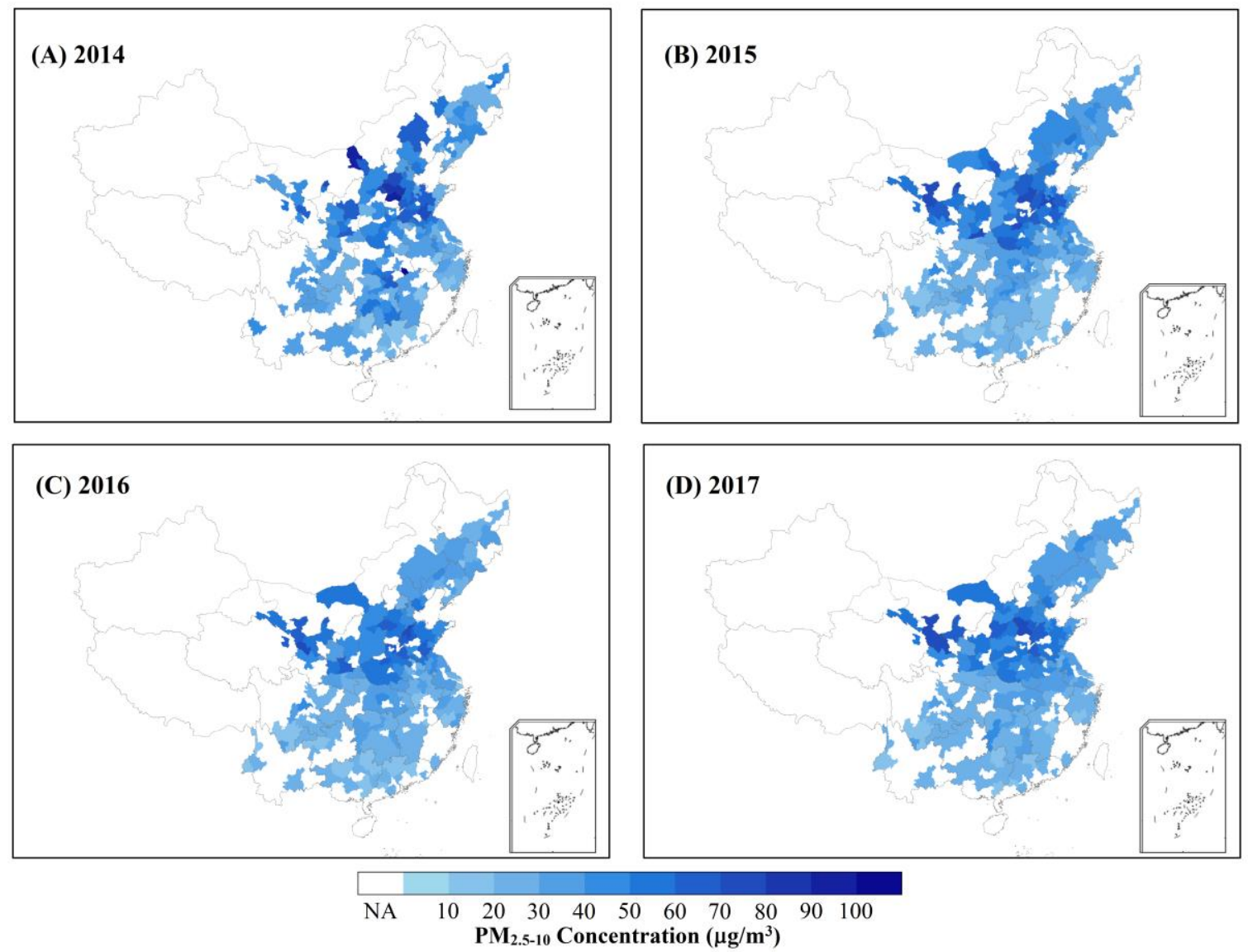

Figure S7. The daily average concentrations of $\mathrm{PM}_{2.5-10}$ in 205 cities from 2014 to 2017, China. NA: not available. 

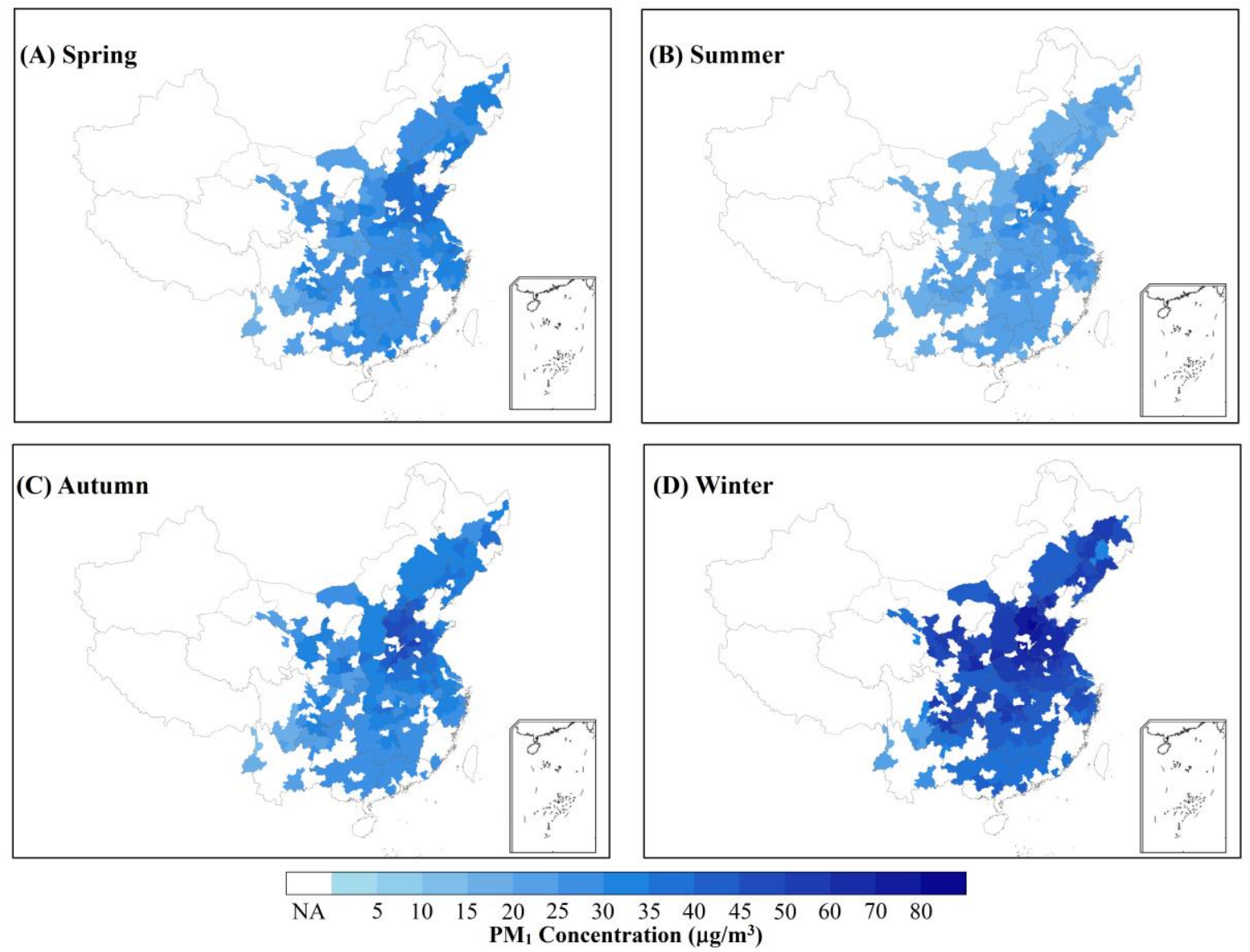

Figure S8. The seasonal average concentrations of $\mathrm{PM}_{1}$ in 205 cities during 2014-2017, China. NA: not available. 

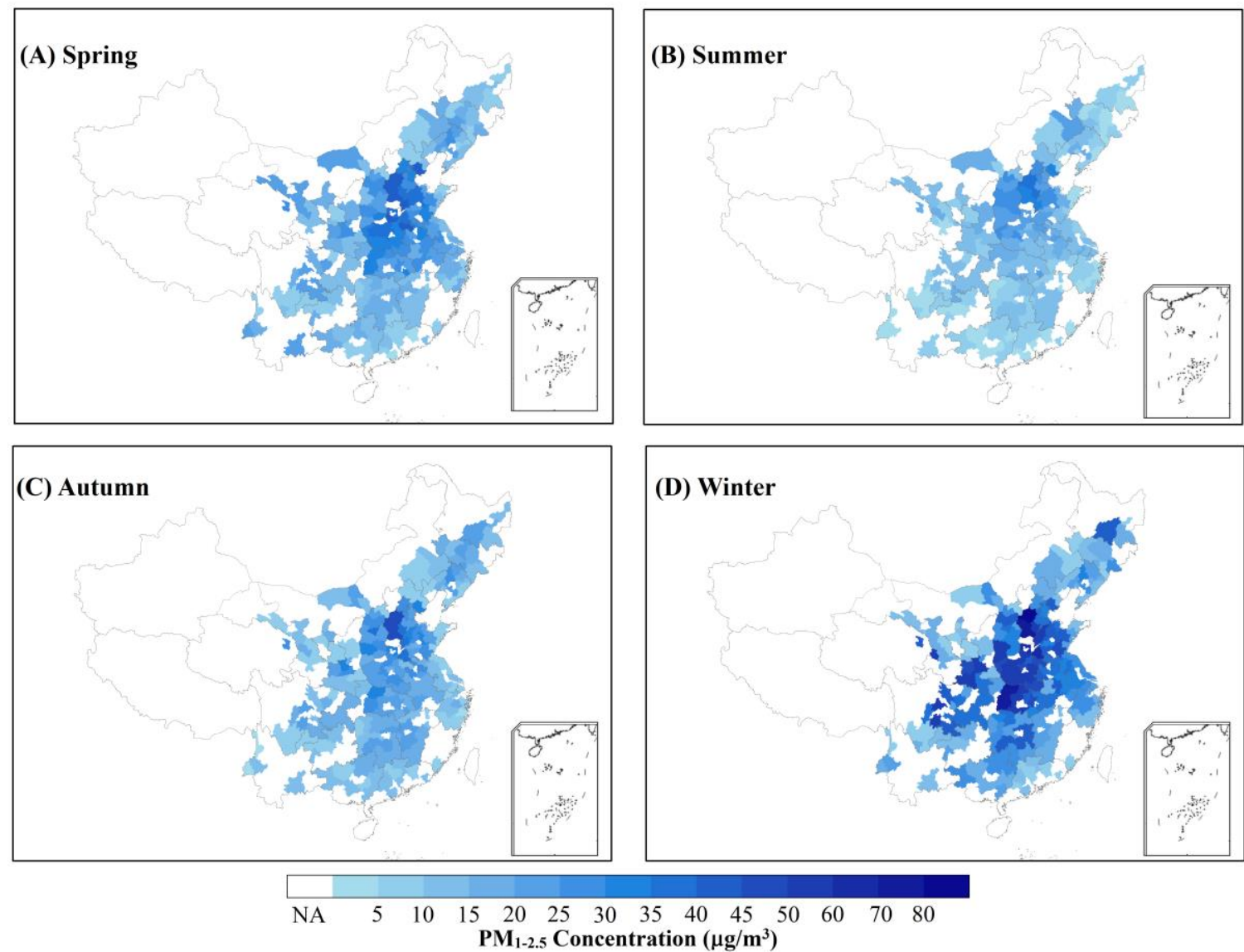

Figure S9. The seasonal average concentrations of $\mathrm{PM}_{1-2.5}$ in 205 cities during 2014-2017, China. NA: not available. 

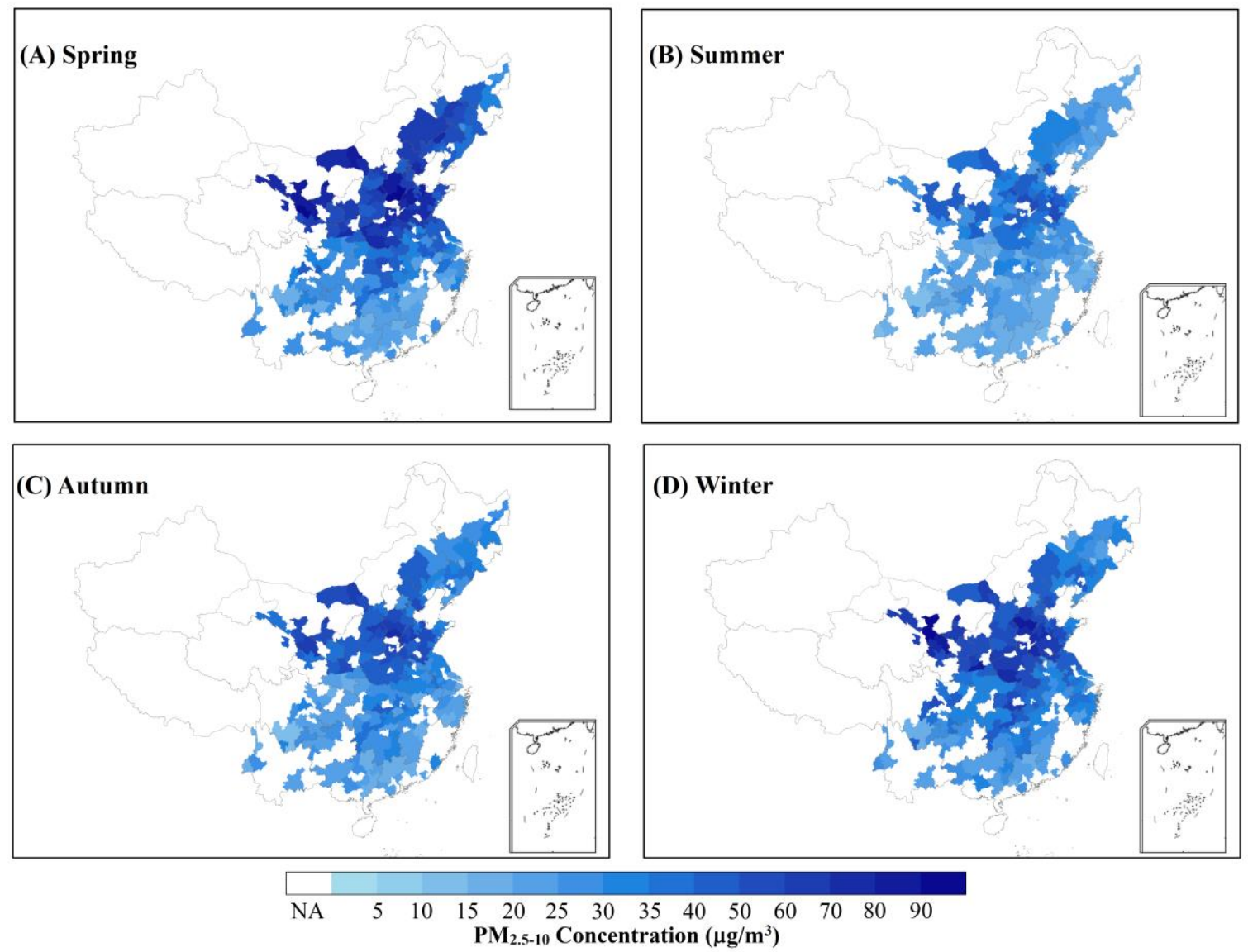

Figure S10. The seasonal average concentrations of $\mathrm{PM}_{2.5-10}$ in 205 cities during 2014-2017, China. NA: not available. 


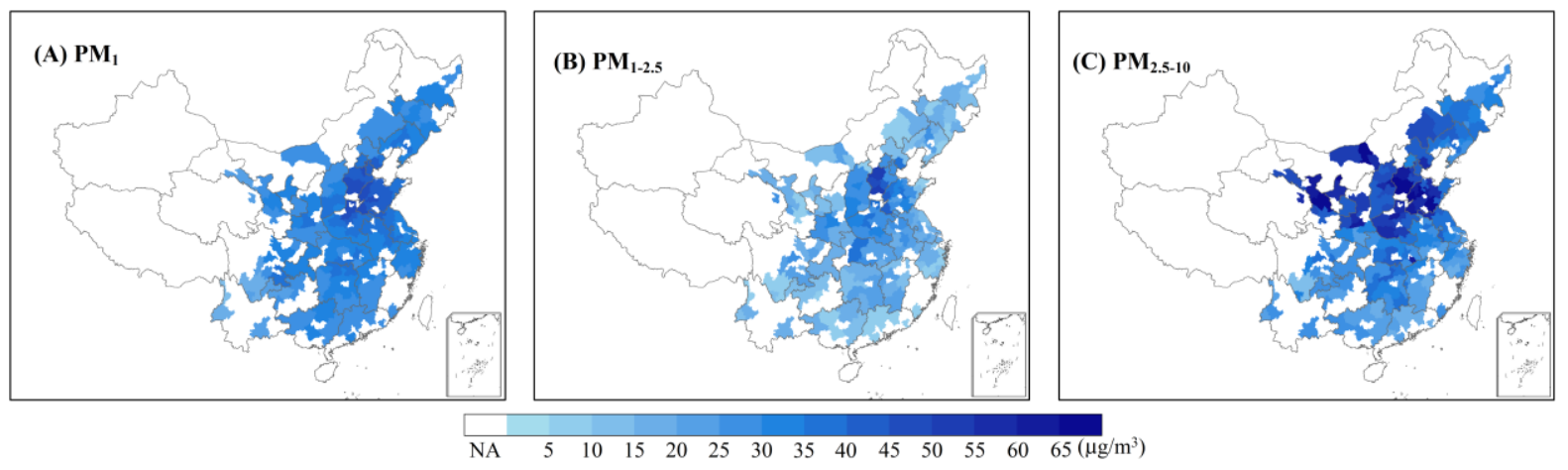

Figure S11. The daily average concentrations of PM2.5-10 in 205 cities during 2014-2017, China. NA: not available. 


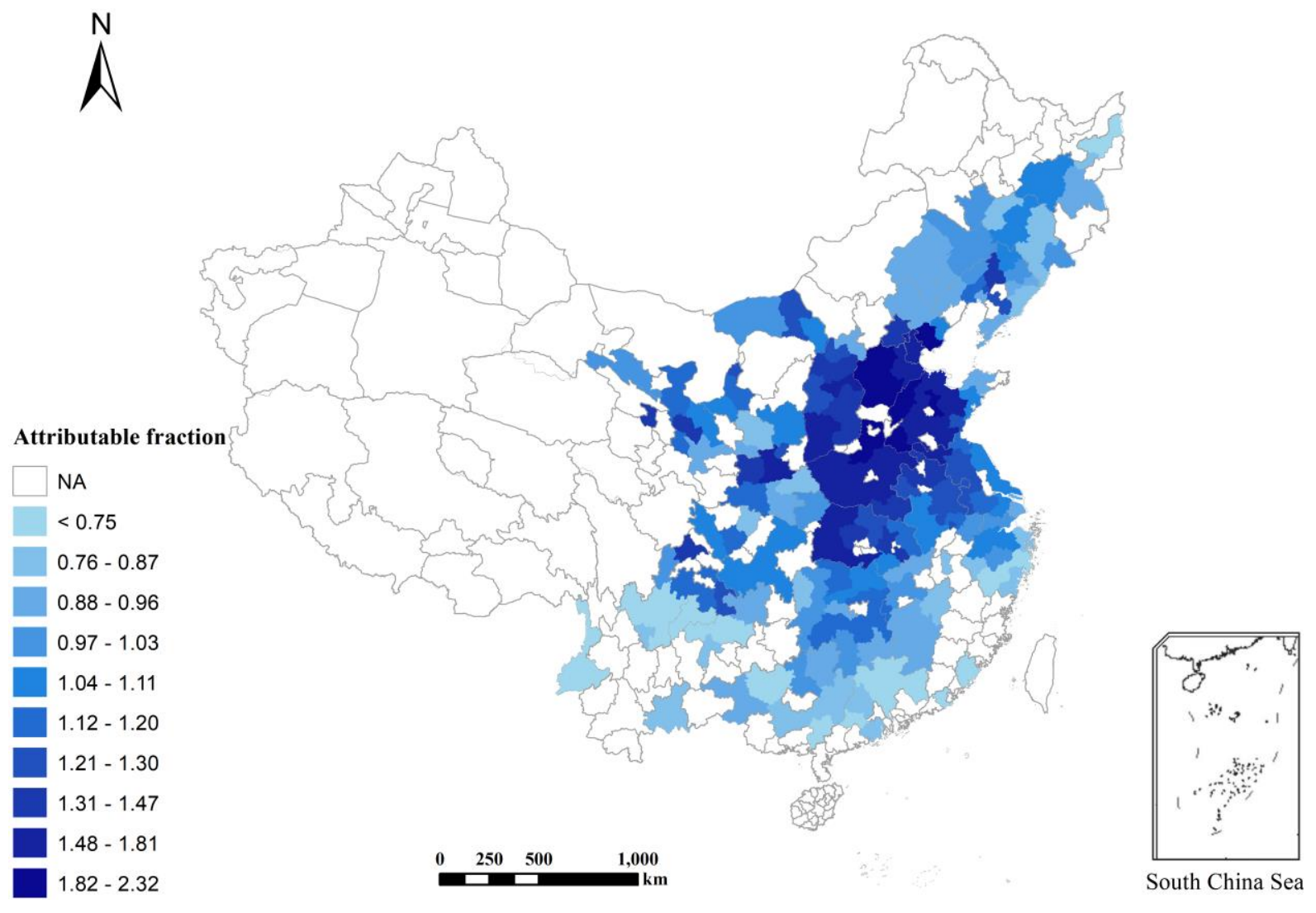

Figure S12. Spatial distributions of attributable fractions (\%) of mortality risk due to particulate matter exposure for 205 cities, China. NA: not available. 


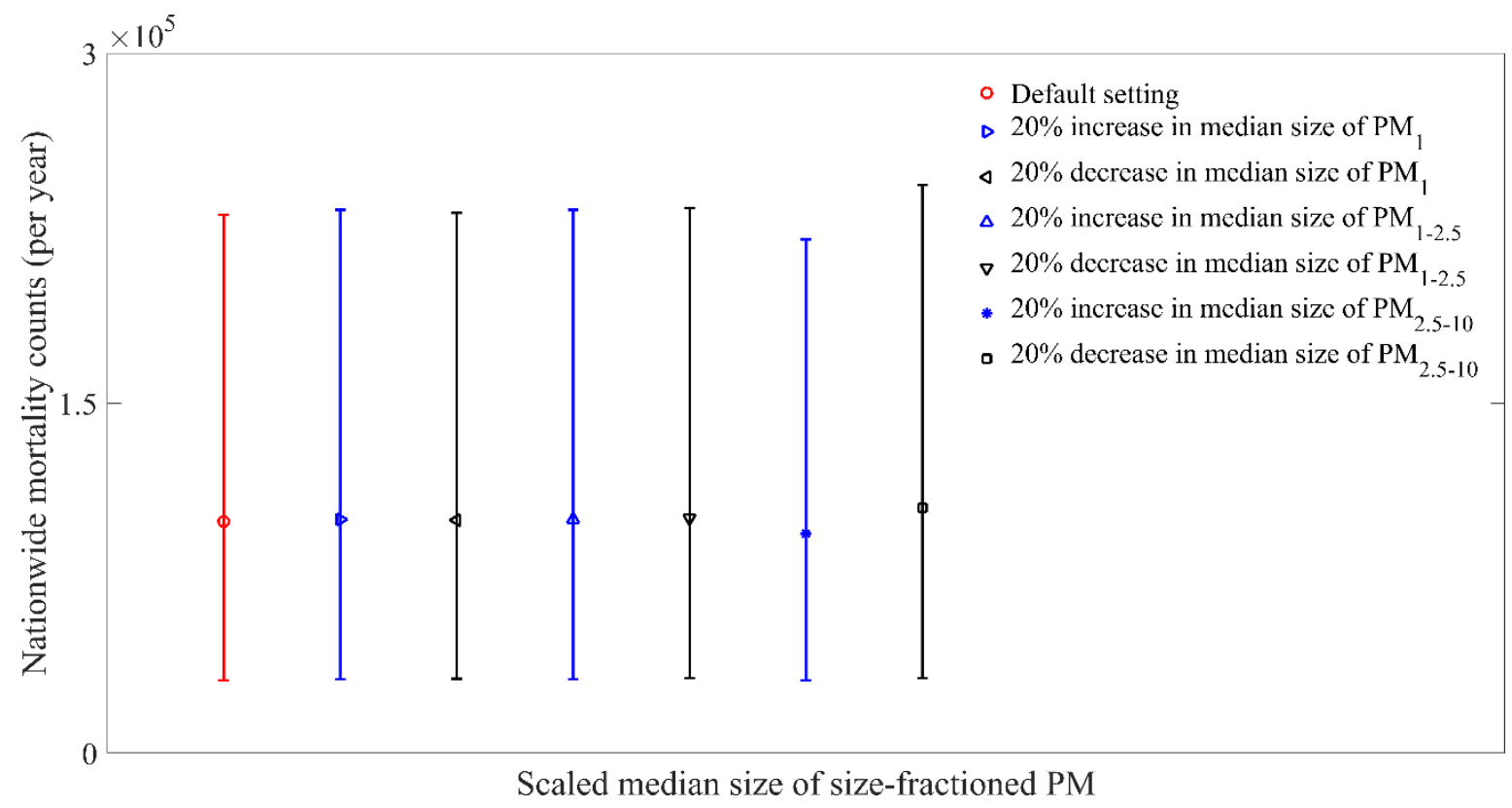

Figure S13. The nationwide mortality counts based on alternatives of changed median size in $\mathrm{PM}_{1}, \mathrm{PM}_{1-2.5}$ and $\mathrm{PM}_{2.5-10}$. For example, $20 \%$ increase in median size of $\mathrm{PM}_{1}$ indicated the median size of $\mathrm{PM}_{1}$ was changed from $577 \mathrm{~nm}$ to $692 \mathrm{~nm}(577 \mathrm{~nm}$ *1.2), given the original setting for median size in $\mathrm{PM}_{1}, \mathrm{PM}_{1-2.5}, \mathrm{PM}_{2.5-10}$ was $577 \mathrm{~nm}, 1885 \mathrm{~nm}$ and $6181 \mathrm{~nm}$, respectively.

Note: PM, particulate matter. 


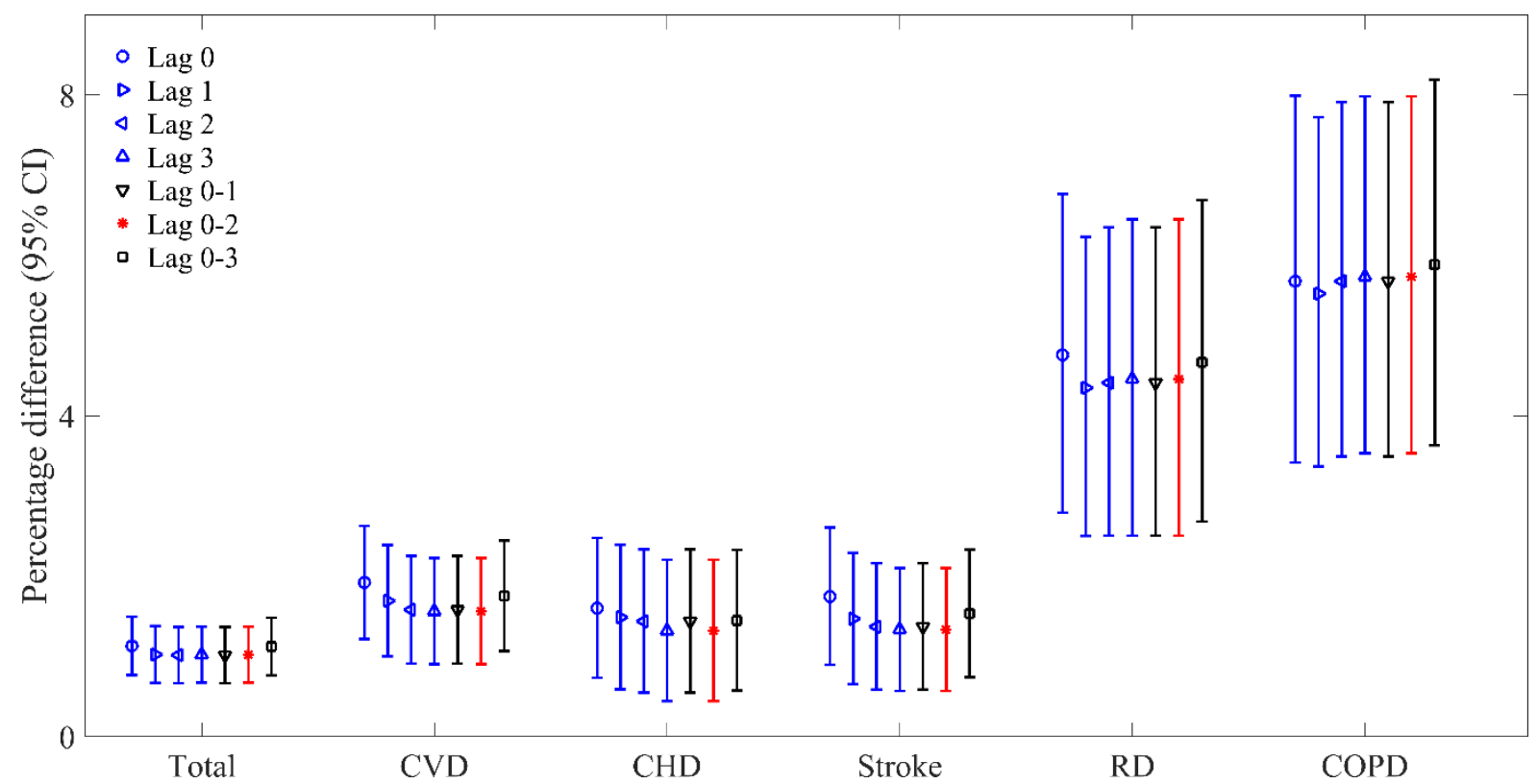

Figure S14. The associations of deposited PM concentrations with cause-specific mortality based on alternate conditions for humidity. Lag $0,1,2,3$ represent the $0 \mathrm{~d}, 1 \mathrm{~d}, 2 \mathrm{~d}$ and $3 \mathrm{~d}$ lags of humidity, and Lag 0-1, 0-2, 0-3 are $2 \mathrm{~d}, 3 \mathrm{~d}$ and $4 \mathrm{~d}$ moving average of humidity.

Note: PM, particulate matter; CVD, cardiovascular disease; CHD, coronary heart disease; RD, respiratory disease; COPD, chronic obstructive pulmonary disease. 


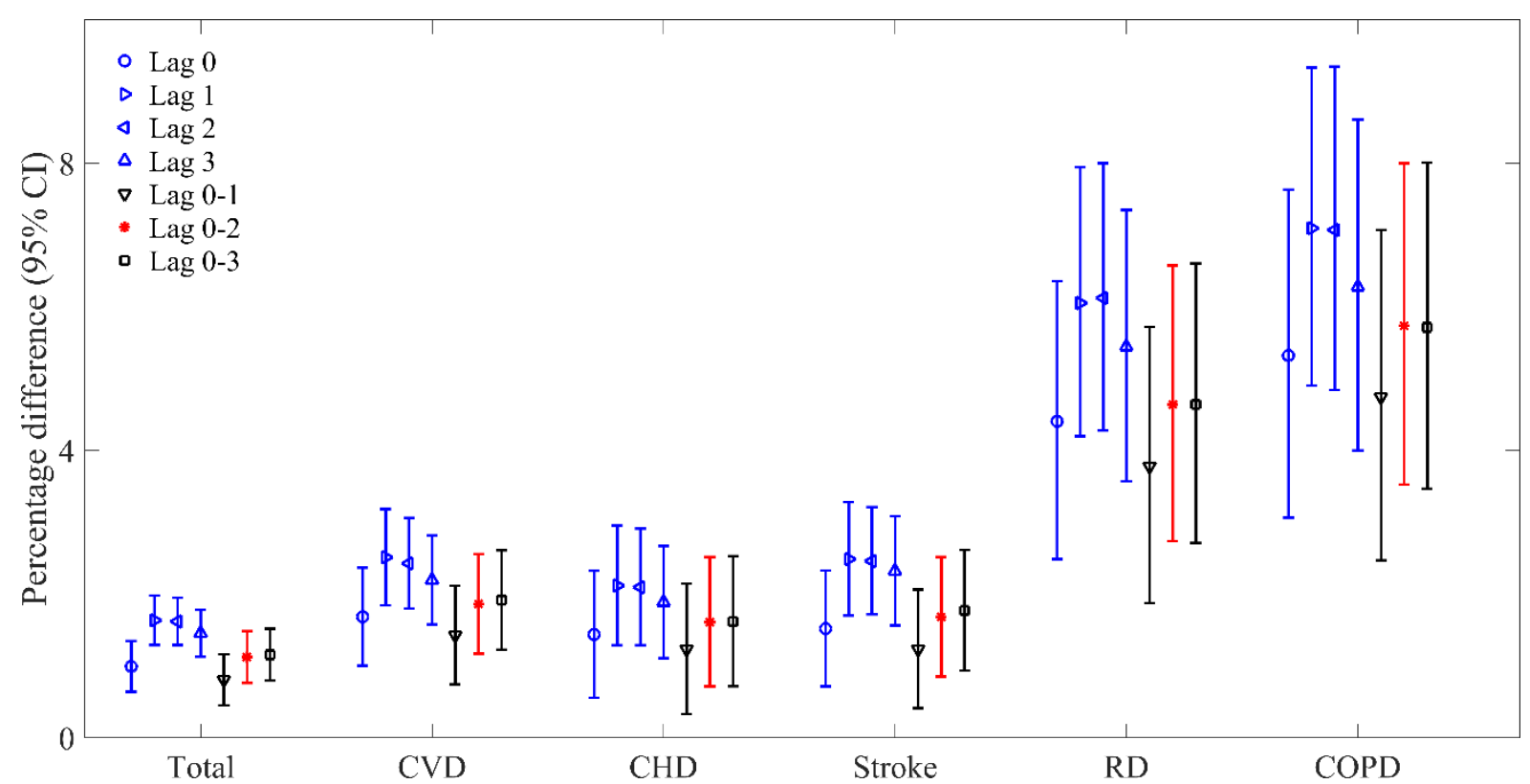

Figure S15. The associations of deposited PM concentrations with cause-specific mortality based on alternate conditions for temperature. Lag $0,1,2,3$ represent the $0 \mathrm{~d}, 1 \mathrm{~d}, 2 \mathrm{~d}$ and $3 \mathrm{~d}$ lags of humidity, and Lag $0-1,0-2,0-3$ are $2 \mathrm{~d}, 3 \mathrm{~d}$ and $4 \mathrm{~d}$ moving average of temperature. Note: PM, particulate matter; CVD, cardiovascular disease; CHD, coronary heart disease; RD, respiratory disease; COPD, chronic obstructive pulmonary disease. 


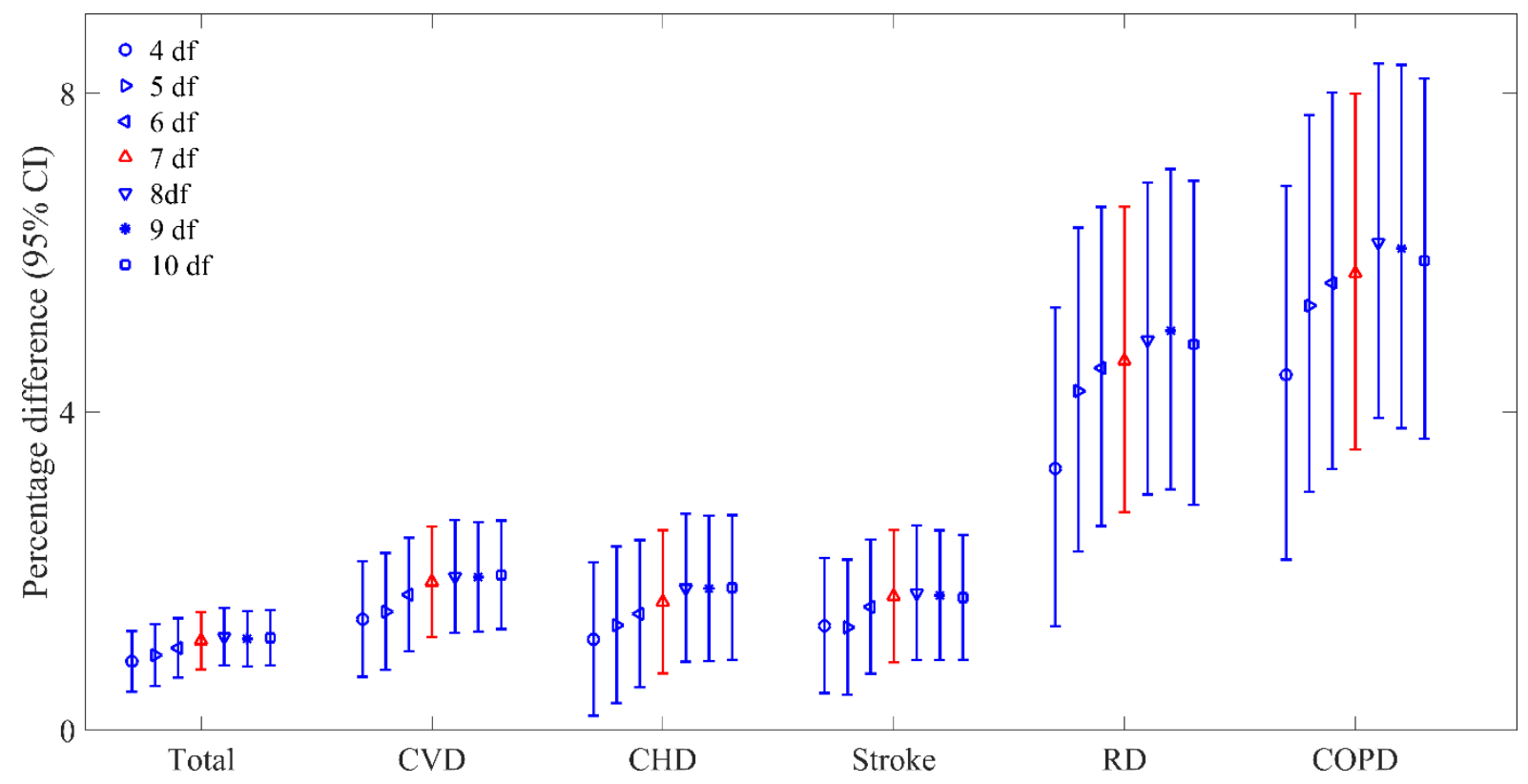

Figure S16. The associations of deposited $\mathrm{PM}_{2.5}$ concentrations with cause-specific mortality based on alternate conditions for the degree freedom of calendar year. $n d f$ represents $n$ degrees of freedom per year used in a natural spline smooth function of calendar day.

Note: PM, particulate matter; CVD, cardiovascular disease; CHD, coronary heart disease; RD, respiratory disease; COPD, chronic obstructive pulmonary disease. 


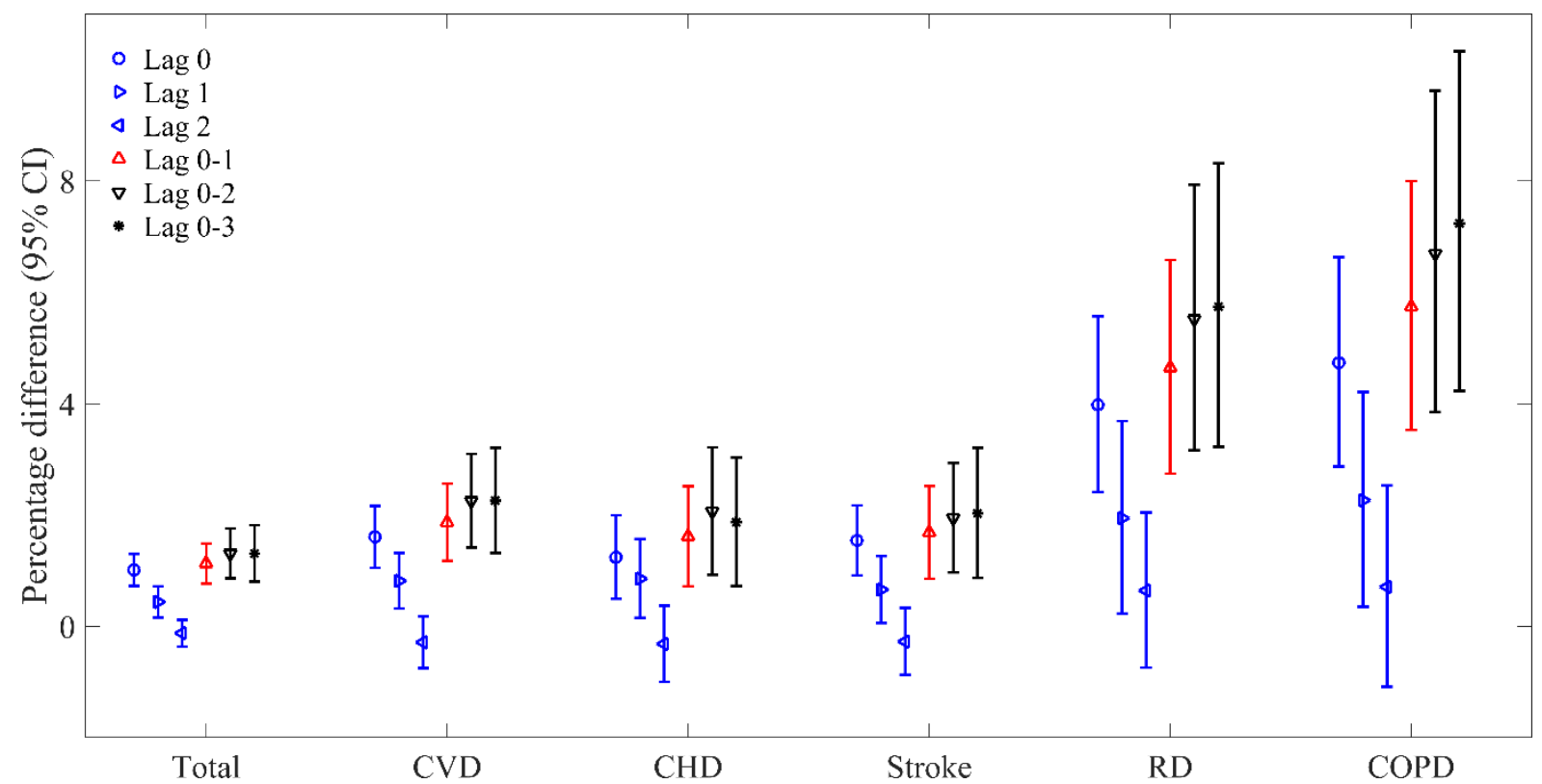

Figure S17. National average percentage difference (posterior mean and 95\% confidence intervals) in daily cause-specific mortality per $10 \mu \mathrm{g} / \mathrm{m}^{3}$ increase in deposited PM concentration exposure in 205 Chinese cities during single-day lags (lag 0,1,2), multiple-day moving average (lag $0-1,0-2,0-3$ represent $2 \mathrm{~d}, 3 \mathrm{~d}$ and $4 \mathrm{~d}$ moving average of deposited PM exposure concentrations).

Note: PM, particulate matter; CVD, cardiovascular disease; CHD, coronary heart disease; RD, respiratory disease; COPD, chronic obstructive pulmonary disease. 


\begin{tabular}{|c|c|c|c|c|c|c|}
\hline $\begin{array}{r}\text { Total Deposited } \\
\text { PM }\end{array}$ & 1 & & & & & \\
\hline $\mathrm{PM}_{2.5-10}$ & 0.91 & 1 & & & & \\
\hline $\mathrm{PM}_{1-2.5}$ & 0.79 & 0.88 & 1 & & & \\
\hline $\mathrm{PM}_{10}$ & 0.63 & 0.89 & 0.8 & 1 & & \\
\hline $\mathrm{PM}_{2.5}$ & 0.38 & 0.44 & 0.82 & 0.42 & 1 & \\
\hline \multirow[t]{2}{*}{$\mathrm{PM}_{1}$} & 0.78 & 0.91 & 0.99 & 0.86 & 0.77 & 1 \\
\hline & $\mathrm{PM}_{1}$ & $\mathrm{PM}_{2.5}$ & $\mathrm{PM}_{10}$ & $\mathrm{PM}_{1-2.5}$ & $\mathrm{PM}_{2.5-10}$ & $\begin{array}{l}\text { Total Deposited } \\
\text { PM }\end{array}$ \\
\hline
\end{tabular}

Figure S18. The correlation matrix between different sizes of particulate matter. Note: PM, particulate matter. 


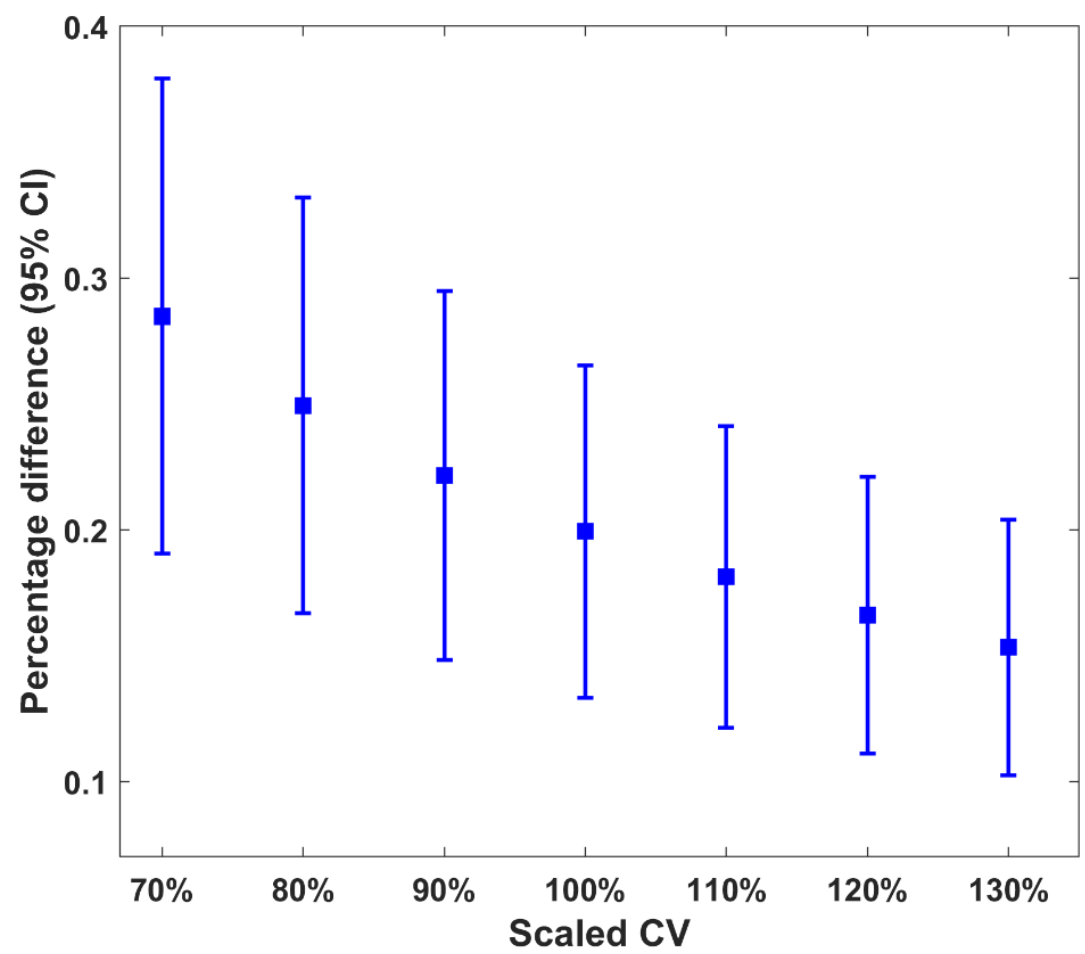

Figure S19. Relationships between scaled CV (coefficient of variation) and percentage differences in daily all-causes mortality per $10 \mu \mathrm{g} / \mathrm{m}^{3}$ increment in $\mathrm{PM}_{2.5}$. The scaled CV was defined in Equation (11) in the manuscript. 
Table S1. Parameters defaulted by multiple-path particle dosimetry model.

\begin{tabular}{lll}
\hline Module variables & & Value \\
\hline \multirow{4}{*}{ Particle Properties } & Aspect Ratio & 1.0 \\
& GSD (diam.) & $1.0 \mu \mathrm{m}$ \\
& GSD (length) & $1.0 \mu \mathrm{m}$ \\
& Correlation & 0.0 \\
\hline \multirow{5}{*}{ Exposure Scenario } & Exposure Condition & Constant exposure \\
& Acceleration of Gravity & $981.0 \mathrm{~cm} / \mathrm{s}^{2}$ \\
& Body Orientation & upright \\
& Inspiratory Fraction & 0.5 \\
& Pause Fraction & 0.0 \\
& Deposition/Clearance & Deposition only \\
\hline
\end{tabular}

Abbreviations: GSD, Geometric Standard Deviation; diam., diameter. 
Table S2. The summary statistics of regional daily PM concentration in 205 Chinese cities, 20142017.

\begin{tabular}{lllllllll}
\hline Variables & Region & Mean & Median & SD & $\mathrm{P}_{25}$ & $\mathrm{P}_{75}$ & Lb_CI & Ub_CI \\
\hline \multirow{2}{*}{$\mathrm{PM}_{1}$} & Nationwide & 33 & 27 & 21 & 17 & 42 & 8 & 95 \\
$\left(\mu \mathrm{g} / \mathrm{m}^{3}\right)$ & South & 31 & 27 & 18 & 18 & 39 & 9 & 77 \\
& North & 36 & 27 & 27 & 18 & 45 & 8 & 109 \\
\hline \multirow{2}{*}{$\mathrm{PM}_{2.5}$} & Nationwide & 54 & 43 & 42 & 28 & 67 & 12 & 161 \\
$\left(\mu \mathrm{g} / \mathrm{m}^{3}\right)$ & South & 48 & 40 & 32 & 26 & 61 & 12 & 130 \\
& North & 60 & 46 & 47 & 29 & 74 & 13 & 186 \\
\hline \multirow{2}{*}{$\mathrm{PM}_{10}$} & Nationwide & 93 & 77 & 63 & 51 & 116 & 24 & 251 \\
$\left(\mu \mathrm{g} / \mathrm{m}^{3}\right)$ & South & 76 & 66 & 45 & 44 & 96 & 22 & 188 \\
& North & 108 & 91 & 73 & 60 & 135 & 28 & 289 \\
\hline
\end{tabular}

Note: Lb_CI: lower bound of 95\%; Ub_CI: upper bound of 95\%; $\mathrm{P}_{25}, 25 \%$ percentage; $\mathrm{P}_{75}, 75 \%$ percentage. 
Table S3. The estimation of excess risk (95\% confidence interval, unit: \%) in daily cause-specific per $10 \mu \mathrm{g} / \mathrm{m}^{3}$ increase in two-day moving average concentration of different exposure metrics.

\begin{tabular}{|c|c|c|c|c|c|c|}
\hline Dosimetry & All-cause & CVD & $\mathrm{CHD}$ & Stroke & $\mathrm{RD}$ & COPD \\
\hline $\mathrm{PM}_{1}$ & $0.35(0.24-0.46)$ & $0.38(0.25-0.51)$ & $0.37(0.19-0.56)$ & $0.32(0.16-0.49)$ & $0.57(0.35-0.80)$ & $0.67(0.41-0.93)$ \\
\hline $\mathrm{PM}_{2.5}$ & $0.20(0.13-0.27)$ & $0.22(0.14-0.30)$ & $0.20(0.09-0.31)$ & $0.19(0.09-0.29)$ & $0.29(0.16-0.43)$ & $0.37(0.21-0.52)$ \\
\hline $\mathrm{PM}_{10}$ & $0.15(0.11-0.20)$ & $0.17(0.11-0.22)$ & $0.15(0.08-0.22)$ & $0.16(0.09-0.23)$ & $0.22(0.13-0.30)$ & $0.26(0.17-0.36)$ \\
\hline $\mathrm{PM}_{2.5-10}$ & $0.26(0.17-0.35)$ & $0.29(0.17-0.40)$ & $0.24(0.10-0.39)$ & $0.30(0.17-0.43)$ & $0.36(0.18-0.54)$ & $0.43(0.23-0.64)$ \\
\hline $\mathrm{PM}_{1-2.5}$ & $0.28(0.14-0.42)$ & $0.34(0.17-0.51)$ & $0.27(0.06-0.48)$ & $0.31(0.12-0.50)$ & $0.37(0.08-0.66)$ & $0.54(0.19-0.89)$ \\
\hline Size-combined PM & $1.12(0.77-1.49)$ & $1.86(1.17-2.56)$ & $1.61(0.72-2.51)$ & $1.68(0.85-2.52)$ & $4.64(2.74-6.58)$ & $5.74(3.52-8.00)$ \\
\hline
\end{tabular}

Abbreviations: All-cause: non-accidental causes; CVD: cardiovascular diseases; CHD: coronary heart disease; RD: respiratory disease; COPD: chronic obstructive pulmonary disease; $\mathrm{PM}_{1}$ : particulate matter with an aerodynamic diameter less than or equal to $1 \mu \mathrm{m}$; $\mathrm{PM}_{2.5}$ : particulate matter with an aerodynamic diameter less than or equal to $2.5 \mu \mathrm{m}$; PM10: particulate matter with an aerodynamic diameter less than or equal to $10 \mu \mathrm{m}$;

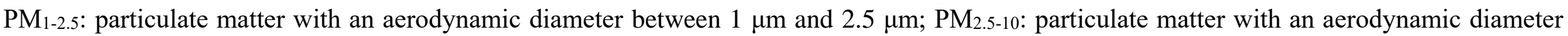
between $2.5 \mu \mathrm{m}$ and $10 \mu \mathrm{m}$. 
Table S4. The estimation of attributable fraction (95\% confidence interval, unit: \%) based on different choices of dosimetry estimation.

\begin{tabular}{lllllll}
\hline Dosimetry & All-cause & CVD & CHD & Stroke & RD & COPD \\
\hline $\mathrm{PM}_{1}$ & $1.16(0.80,1.51)$ & $1.24(0.81,1.68)$ & $1.22(0.61,1.83)$ & $1.07(0.52,1.62)$ & $1.90(1.14,2.66)$ & $2.20(1.34,3.08)$ \\
$\mathrm{PM}_{2.5}$ & $1.05(0.70,1.41)$ & $1.16(0.74,1.58)$ & $1.06(0.50,1.63)$ & $1.01(0.50,1.52)$ & $1.56(0.84,2.28)$ & $1.95(1.12,2.78)$ \\
$\mathrm{PM}_{10}$ & $1.39(0.98,1.79)$ & $1.54(1.03,2.03)$ & $1.36(0.71,2.02)$ & $1.45(0.84,2.07)$ & $1.97(1.18,2.75)$ & $2.40(1.50,3.32)$ \\
$\mathrm{PM}_{2.5-10}$ & $0.97(0.64,1.31)$ & $1.07(0.65,1.50)$ & $0.92(0.38,1.46)$ & $1.13(0.63,1.62)$ & $1.35(0.66,2.05)$ & $1.63(0.85,2.41)$ \\
$\mathrm{PM}_{1-2.5}$ & $0.57(0.29,0.85)$ & $0.68(0.33,1.02)$ & $0.54(0.11,0.97)$ & $0.62(0.24,1.00)$ & $0.74(0.16,1.31)$ & $1.08(0.39,1.79)$ \\
Size-combined PM & $1.18(0.80,1.56)$ & $1.17(0.74,1.6)$ & $1.01(0.45,1.58)$ & $1.05(0.53,1.58)$ & $1.94(1.15,2.75)$ & $2.41(1.47,3.35)$ \\
\hline
\end{tabular}

Abbreviations: All-cause: non-accidental causes; CVD: cardiovascular diseases; CHD: coronary heart disease; RD: respiratory disease; COPD: chronic obstructive pulmonary disease; $\mathrm{PM}_{1}$ : particulate matter with an aerodynamic diameter less than or equal to $1 \mu \mathrm{m}$; $\mathrm{PM}_{2.5}$ : particulate matter with an aerodynamic diameter less than or equal to $2.5 \mu \mathrm{m}$; $\mathrm{PM}_{10}$ : particulate matter with an aerodynamic diameter less than or equal to $10 \mu \mathrm{m}$; PM1-2.5: particulate matter with an aerodynamic diameter between $1 \mu \mathrm{m}$ and $2.5 \mu \mathrm{m}$; PM2.5-10: particulate matter with an aerodynamic diameter between $2.5 \mu \mathrm{m}$ and $10 \mu \mathrm{m}$. 
Table S5. The estimated mortality counts when setting a threshold of $5 \mu \mathrm{g} / \mathrm{m}^{3}$ for $\mathrm{PM}_{2.5}$

\begin{tabular}{ccccc}
\hline Disease & $\begin{array}{c}\text { Mortality } \\
\text { Counts }\end{array}$ & $95 \%$ CI & $\begin{array}{c}\text { Differences of estimated } \\
\text { mortality }\end{array}$ & $\begin{array}{c}\text { Change ratio } \\
(\%)\end{array}$ \\
\hline All-cause & 81042 & $54263 \sim 107724$ & -7866 & -8.85 \\
CVD & 45302 & $29025 \sim 61505$ & -4281 & -8.63 \\
CHD & 16480 & $7770 \sim 25219$ & -1521 & -8.45 \\
Stroke & 18937 & $9394 \sim 28429$ & -1816 & -8.75 \\
RD & 14001 & $7624 \sim 20333$ & -1435 & -9.29 \\
COPD & 13374 & $7778 \sim 18962$ & -1364 & -9.26 \\
\hline
\end{tabular}

Note. CI: confidence interval; All-cause: non-accidental causes; CVD: cardiovascular diseases; CHD: coronary heart disease; RD: respiratory disease; COPD: chronic obstructive pulmonary disease. 